\title{
Microscopic analogue of the Landau free energy for three-dimensional Ising-like systems
}

\author{
M.P.Kozlovskii, I.V.Pylyuk, O.O.Prytula \\ Institute for Condensed Matter Physics \\ of the National Academy of Sciences of Ukraine, \\ 1 Svientsitskii Str., 79011 Lviv, Ukraine
}

Received October 14, 2002, in final form February 12, 2003

\begin{abstract}
A microscopic analogue of the Landau free energy for a three-dimensional one-component spin system is found below the critical temperature as a result of direct calculations. The obtained explicit expressions make it possible to analyse the dependence of coefficients of the analogue on temperature and microscopic parameters of the system. In contrast to the case for the Landau theory, the temperature dependence of these coefficients is nonanalytic. The quantities determining the coefficients in the expression for a microscopic analogue of the Landau free energy as well as the temperature-dependence curves for the order parameter of the system are given for different values of the effective radius of the exponentially decreasing interaction potential.
\end{abstract}

Key words: Ising-like system, collective variables, Landau free energy, order parameter

PACS: $05.50 .+q, 64.60 . F r, 75.10 . H k$

\section{Object of investigation and method}

We consider a three-dimensional (3D) Ising-like system on a simple cubic lattice with period $c$. The interaction potential is an exponentially decreasing function $\Phi\left(r_{\mathbf{j} \mathbf{1}}\right)=A \exp \left(-r_{\mathbf{j} \mathbf{1}} / b\right)$. Here, $A$ is a constant, $r_{\mathbf{j} \mathbf{1}}$ is the interparticle distance, and $b$ is the radius of effective interaction. The approximation for the Fourier transform of the interaction potential is taken in the form [1]

$$
\tilde{\Phi}(k)= \begin{cases}\tilde{\Phi}(0)\left(1-2 b^{2} k^{2}\right), & k \leqslant B^{\prime} \\ 0, & B^{\prime}<k \leqslant B\end{cases}
$$

where $B$ is the boundary of Brillouin half-zone $(B=\pi / c), B^{\prime}=(b \sqrt{2})^{-1}, \tilde{\Phi}(0)=$ $8 \pi A(b / c)^{3}$. 
A microscopic analogue of the Landau free energy for 3D Ising-like systems is calculated in the present paper using the collective variables (CV) method (see [1]).

The CV method allows us to calculate the partition function of the system and to obtain not only the universal quantities (critical exponents) but also the nonuniversal characteristics. The methods existing at present make it possible to calculate universal quantities to a quite high degree of accuracy (see, for example, [2-5]). The advantage of the proposed method is the possibility of deriving analytic expressions for the phase transition temperature and the amplitudes of thermodynamic characteristics as functions of microscopic parameters of the initial system (the lattice constant and parameters of the interaction potential), which makes this method useful in describing the phase transitions in a wide class of 3D systems.

The term collective variables is applied to a special class of variables specific for each individual physical system. The set of CV contains variables associated with order parameters. For this reason, the phase space of $\mathrm{CV}$ is the most natural one to use for describing a phase transition. For magnetic systems, the CV $\rho_{\mathbf{k}}$ are the variables associated with modes of spin moment density oscillations, while the order parameter is associated with the variable $\rho_{0}$, in which the subscript " 0 " corresponds to the peak of the Fourier transform of the interaction potential. An important factor in describing the system behaviour near the phase transition temperature $T_{\mathrm{c}}$ by the CV method is the use of non-Gaussian measure densities. A non-Gaussian density of measure at a zero external field is represented as an exponential function of the $\mathrm{CV}$, the argument of which contains, along with the quadratic term, even higher powers of the variable with the corresponding coupling constants. The simplest nonGaussian measure density is the quartic one (the $\rho^{4}$ model) with the second and the fourth powers of the variable in the exponent. The sextic measure density (the $\rho^{6}$ model) includes the sixth power of the variable in addition to the second and the fourth powers, etc. The quartic approximaton allows us to describe all qualitative aspects of the second-order phase transition, while the sextic approximaton ensures a more adequate quantitative description of the critical properties of a spin system. This is confirmed by calculations as well as by the analysis of the behaviour of the coefficients in the initial expression for partition function and the critical exponent of the correlation length for the sequence of $\rho^{4}, \rho^{6}, \rho^{8}$, and $\rho^{10}$ models $[1,6-8]$ as well as by the calculation and comparison of thermodynamic functions for the models $\rho^{4}$ and $\rho^{6}[9]$ and by comparison of the results of our calculations with other available data (see, for example, $[9,10]$ ).

In this paper, the critical behaviour of a 3D Ising-like system is studied on the basis of the higher non-Gaussian approximation (the $\rho^{6}$ model) taking into account the correction-to-scaling terms.

The starting point of the problem statement in the CV method is the Hamiltonian of a 3D Ising-like system. After passing to the CV set, the Jacobian of the transition from the spin variables to the CV is calculated to obtain a partition function functional similar to the Ginzburg-Landau functional. The partition function of the spin system is integrated over the layers of the CV phase space. The corresponding renormalization group $(\mathrm{RG})$ transformation can be related to the Wilson type. 
Although the CV method like the Wilson approach exploits the RG ideas, it is based on the use of a non-Gaussian density of measure. The main feature is the integration of short-wave spin density oscillation modes, which is generally done without using perturbation theory. For this purpose, we divide the phase space of the $\mathrm{CV} \rho_{\mathbf{k}}$ into layers with the division parameter $s$. In each $n$-th layer (corresponding to the region of wave vectors $B_{n+1}<k \leqslant B_{n}, B_{n+1}=B_{n} / s, s>1$ ), the Fourier transform of the potential $\tilde{\Phi}(k)$ is replaced by its average value (the arithmetic mean in the given case). To simplify the presentation, we assume that the correction for the potential averaging is zero, although it can be taken into account if necessary [1]. Including this correction leads to a nonzero value of the critical exponent $\eta$ characterizing the behaviour of the pair correlation function for $T=T_{\mathrm{c}}$. As a result of step-by-step calculation of partition function, the number of integration variables in the expression for this quantity decreases gradually. The partition function is then represented as a product of partial partition functions $Q_{n}$ of separate layers and the integral of the "smoothed" effective measure density $\mathcal{W}_{6}^{(n+1)}(\rho)$ :

$$
Z=2^{N} 2^{\left(N_{n+1}-1\right) / 2} Q_{0} Q_{1} \cdots Q_{n}\left[Q\left(P_{n}\right)\right]^{N_{n+1}} \int \mathcal{W}_{6}^{(n+1)}(\rho)(\mathrm{d} \rho)^{N_{n+1}}
$$

Here $N_{n+1}=N^{\prime} s^{-3(n+1)}, N^{\prime}=N s_{0}^{-3}, s_{0}=B / B^{\prime}=\pi \sqrt{2} b / c$. The sextic density of measure of the $(n+1)$ th block structure $\mathcal{W}_{6}^{(n+1)}(\rho)$ has the form

$$
\begin{aligned}
\mathcal{W}_{6}^{(n+1)}(\rho)=\exp & {\left[-\frac{1}{2} \sum_{k \leqslant B_{n+1}} d_{n+1}(k) \rho_{\mathbf{k}} \rho_{-\mathbf{k}}\right.} \\
& \left.-\sum_{l=2}^{3} \frac{a_{2 l}^{(n+1)}}{(2 l) ! N_{n+1}^{l-1}} \sum_{k_{1}, \ldots, k_{2 l} \leqslant B_{n+1}} \rho_{\mathbf{k}_{1}} \cdots \rho_{\mathbf{k}_{2 l}} \delta_{\mathbf{k}_{1}+\cdots+\mathbf{k}_{2 l}}\right]
\end{aligned}
$$

where $\delta_{\mathbf{k}_{1}+\cdots+\mathbf{k}_{2 l}}$ is the Kronecker symbol, $B_{n+1}=B^{\prime} s^{-(n+1)}, d_{n+1}(k)=a_{2}^{(n+1)}-$ $\beta \tilde{\Phi}(k), \beta=1 /(k T)$. The coefficients $a_{2 l}^{(n+1)}$ are renormalized values of the initial coefficients $a_{2 l}^{\prime}$ after integration over $n+1$ layers of the phase space of $\mathrm{CV}$. The quantities $a_{2 l}^{\prime}$ are determined by special functions $[9,10]$ and are functions of $s_{0}$, i.e., of the ratio $b / c$.

The basic idea of the calculation of explicit expressions for free energy and other thermodynamic functions of the system near $T_{\mathrm{c}}$ on a microscopic level $\left(\tau<\tau^{*} \sim\right.$ $\left.10^{-2}, \tau=\left(T-T_{\mathrm{c}}\right) / T_{\mathrm{c}}\right)$ lies in the separate inclusion of contributions from shortwave and long-wave modes of spin moment density oscillations [1,11]. The shortwave modes are characterized by the presence of RG symmetry and are described by a non-Gaussian measure density. Here, the RG method is used (see, for example, [12]). These modes are responsible for the formation of critical exponents and for renormalization of the coefficient of the distribution describing the long-wave modes. The way in which the contribution from long-wave modes of oscillations to the free energy of the system is taken into account differs qualitatively from the method of calculating the short-wave part of the partition function. The calculation of this 
contribution is based on using the Gaussian density of measure as the basis density. We have developed a direct method of calculation with the results obtained by taking into account the short-wave modes as initial parameters.

Calculating separately the contributions to the free energy from short- and longwave modes of spin density oscillations, we can obtain a complete expression for the free energy of the system. Detailed calculations of the contributions to the free energy of the system from short- and long-wave modes and the coefficients of complete expressions for the entropy, internal energy, specific heat are presented in [13,14]. A calculation technique for the first correction to scaling is elaborated in the course of determining the thermodynamic functions of the system in the $\rho^{6}$ model approximation. It is shown that each of the leading critical amplitudes and confluent correction amplitudes can be represented as a product of a universal factor not depending on the microscopic parameters of the system and a nonuniversal factor depending on these parameters.

The suggested approach makes it possible to investigate the dependences of thermodynamic characteristics of a 3D Ising-like system on its microscopic parameters [15].

\section{Microscopic analogue of the Landau free energy and order parameter of a 3D Ising-like system}

The role of the order parameter for the system under investigation is played by the average spin moment. It is associated with the existence of a nonzero value $\bar{\rho}_{0}$ below the phase transition temperature, for which the integrand of the expression for the long-wave part of the partition function

$$
Z_{\mu_{\tau}+1}=\mathrm{e}^{-\beta F_{\mu_{\tau}+1}^{\prime}} \int \exp \left[\beta \sqrt{N} \rho_{0} h+\tilde{B} \rho_{0}^{2}-\frac{G}{N} \rho_{0}^{4}-\frac{D}{N^{2}} \rho_{0}^{6}\right] \mathrm{d} \rho_{0}
$$

attains its extremum value. Here $\beta$ is the inverse temperature, $h$ is determined by the value of the constant external magnetic field $\mathcal{H}$ introduced in our analysis $\left(h=\mu_{\mathrm{B}} \mathcal{H}\right.$, $\mu_{\mathrm{B}}$ being the Bohr magneton). The expression for $-\beta F_{\mu_{\tau}+1}^{\prime}$ corresponding to the contribution to the free energy of the system from $\mathrm{CV} \rho_{\mathbf{k}}$ with the values of wave vectors $k \rightarrow 0$ (but not equal to zero) as well as the coefficients of the expressions

$$
\begin{aligned}
\tilde{B} & =\tilde{B}^{(0)}|\tau|^{2 \nu} \beta \tilde{\Phi}(0)\left(1+\tilde{B}^{(1)}|\tau|^{\Delta_{1}}\right), \\
G & =G^{(0)}|\tau|^{\nu}(\beta \tilde{\Phi}(0))^{2}\left(1+G^{(1)}|\tau|^{\Delta_{1}}\right), \\
D & =D^{(0)}(\beta \tilde{\Phi}(0))^{3}\left(1+D^{(1)}|\tau|^{\Delta_{1}}\right)
\end{aligned}
$$

are given in $[15,16]$. Here $\nu$ and $\Delta_{1}$ are the critical exponent of the correlation length and the exponent of the first correction to scaling, respectively. Carrying out in (4) the substitution of the variable

$$
\rho_{0}=\sqrt{N} \rho
$$

we obtain

$$
Z_{\mu_{\tau}+1}=\mathrm{e}^{-\beta F_{\mu_{\tau}+1}^{\prime}} \sqrt{N} \int \mathrm{e}^{-N E_{0}(\rho)} \mathrm{d} \rho,
$$


Table 1. Values of quantities determining the coefficients in the expression for a microscopic analogue of the Landau free energy.

\begin{tabular}{crrrrr}
\hline$b$ & \multicolumn{1}{c}{$b_{\mathrm{I}}$} & \multicolumn{1}{c}{$b_{\mathrm{II}}$} & \multicolumn{1}{c}{$b_{\mathrm{III}}$} & \multicolumn{1}{c}{$c$} & \multicolumn{1}{c}{$2 c$} \\
\hline$s=2.0000$ & & & & & \\
$\tilde{B}^{(0)}$ & 1.0106 & 0.9530 & 0.9305 & 0.7258 & 0.7149 \\
$\tilde{B}^{(1)}$ & -0.2733 & -0.3959 & -0.4420 & -0.8188 & -0.8375 \\
$G^{(0)}$ & 0.0550 & 0.0857 & 0.1010 & 1.9382 & 15.3880 \\
$G^{(1)}$ & -0.8919 & -1.2918 & -1.4423 & -2.6720 & -2.7330 \\
$D^{(0)}$ & 0.0009 & 0.0023 & 0.0033 & 1.5614 & 99.9318 \\
$D^{(1)}$ & -0.6952 & -0.9377 & -1.0470 & -1.9396 & -1.9839 \\
$s=2.7349$ & & & & & \\
$\tilde{B}^{(0)}$ & 0.9417 & 0.8888 & 0.8683 & 0.6865 & 0.6768 \\
$\tilde{B}^{(1)}$ & -0.4451 & -0.5124 & -0.5377 & -0.7445 & -0.7550 \\
$G^{(0)}$ & 0.0690 & 0.1074 & 0.1267 & 2.4478 & 19.4434 \\
$G^{(1)}$ & -1.1718 & -1.3491 & -1.4157 & -1.9601 & -1.9876 \\
$D^{(0)}$ & 0.0012 & 0.0031 & 0.0044 & 2.0825 & 133.281 \\
$D^{(1)}$ & -0.8853 & -1.0193 & -1.0696 & -1.4809 & -1.5017 \\
$s=3.0000$ & & & & & \\
$\tilde{B}^{(0)}$ & 0.9115 & 0.8610 & 0.8415 & 0.6697 & 0.6605 \\
$\tilde{B}^{(1)}$ & -0.4755 & -0.5321 & -0.5533 & -0.7261 & -0.7348 \\
$G^{(0)}$ & 0.0732 & 0.1141 & 0.1346 & 2.6087 & 20.7264 \\
$G^{(1)}$ & -1.1967 & -1.3392 & -1.3926 & -1.8275 & -1.8495 \\
$D^{(0)}$ & 0.0013 & 0.0033 & 0.0047 & 2.2185 & 141.986 \\
$D^{(1)}$ & -0.9113 & -1.0199 & -1.0606 & -1.3918 & -1.4085 \\
\hline
\end{tabular}

and the evaluation of the order parameter is reduced to determining the extremum point $\bar{\rho}$ of the expression

$$
E_{0}(\rho)=D \rho^{6}+G \rho^{4}-\tilde{B} \rho^{2}-\beta h \rho
$$

The value of $\bar{\rho}$ coincides with the average value of $\rho$ corresponding to the equilibrium value of the order parameter $[1,9,10]$. The expression for $E_{0}(\rho)$ defines the fraction of free energy associated with the order parameter. It corresponds to a microscopic analogue of the Landau free energy. The quantity $Z_{\mu_{\tau}+1}$ will be expressed in terms of $E_{0}(\bar{\rho})$ (coinciding in form with the expansion of the free energy into a power series in the order parameter) by using the steepest descent method for evaluating the integral (7) (see [16]).

The expression (8) was derived by successive elimination of "insignificant" variables $\rho_{\mathbf{k}}$ with $k \neq 0$, which allowed us to calculate the coefficients of $E_{0}(\rho)$ (see table 1). Numerical values in table 1 are given for some values of the effective radius $b$ of the potential and optimal values of the RG parameter $s$ [9]. The value of $b=b_{\mathrm{I}}=c /(2 \sqrt{3})$ corresponds to the interaction between the nearest neighbours, 
$b=b_{\mathrm{II}}=0.3379 c$ corresponds to the interaction between the nearest and nextnearest neighbours, and $b=b_{\mathrm{III}}=0.3584 c$ corresponds to the nearest, next-nearest, and third neighbours [17]. At these values of $b$ and at small values of the wave vectors $\mathbf{k}$, the parabolic approximation of the Fourier transform of the exponentially decreasing interaction potential corresponds to the analogous approximation of the Fourier transform for the interaction potentials of the above-mentioned neighbours. For $s=s^{*}=2.7349$, the average value of the coefficient in the term with the second power of the variable in the expression for the effective measure density is equal to zero at a fixed point (in the $\rho^{4}$ model, this corresponds to $s^{*}=3.5862$ ). Thus, there is no need to postulate a temperature dependence of the coefficients in equation (8) (as in the case of the Landau expansion) since the analytic form of their dependence on temperature and microscopic parameters of the system has been obtained as a result of direct calculations. Unlike the Landau theory case, the temperature dependence of these coefficients is nonanalytic (see (5)).

Let us go over to direct calculations of the average spin moment. The point $\bar{\rho}$ can be determined from the condition for the extremum $\partial E_{0}(\rho) / \partial \rho=0$ or

$$
6 D \bar{\rho}^{5}+4 G \bar{\rho}^{3}-2 \tilde{B} \bar{\rho}-\frac{h}{k T}=0 .
$$

For $h=0$, we obtain the biquadratic equation

$$
6 D \bar{\rho}^{4}+4 G \bar{\rho}^{2}-2 \tilde{B}=0 .
$$

Solving this equation and separating temperature explicitly, we arrive at the following formula for the average spin moment $\langle\sigma\rangle=\bar{\rho}$ :

$$
\langle\sigma\rangle=\langle\sigma\rangle^{(0)}|\tau|^{\beta}\left(1+\langle\sigma\rangle^{(1)}|\tau|^{\Delta_{1}}\right) .
$$

Here, $\beta=\nu / 2$ is the critical exponent of the average spin moment, and the coefficients $\langle\sigma\rangle^{(l)}$ are given in $[15,16]$. The curves describing the dependence of $\langle\sigma\rangle$ on $\tau$ for various values of $b$ are shown in figure 1 for $s=3$.

\section{Conclusions}

The partition function of a 3D Ising-like system is integrated over the layers of the CV phase space using the sextic measure density. Integration is performed over all variables except for the variable $\rho_{0}$ connected with the order parameter. Partition function is reduced to a single integral. The exponent of the integrand contains the energy $E_{0}(\rho)$ (see $(7)$, (8)), which corresponds to a microscopic analogue of the Landau free energy. The expression for $E_{0}(\rho)$ can be regarded as the part of free energy associated with the order parameter.

The coefficients of a microscopic analogue of the Landau free energy as functions of temperature and microscopic parameters of the system are obtained on the basis of analytic calculations. In contrast to the Landau theory, the dependence of these coefficients on the temperature is nonanalytic. Numerical values of the quantities 


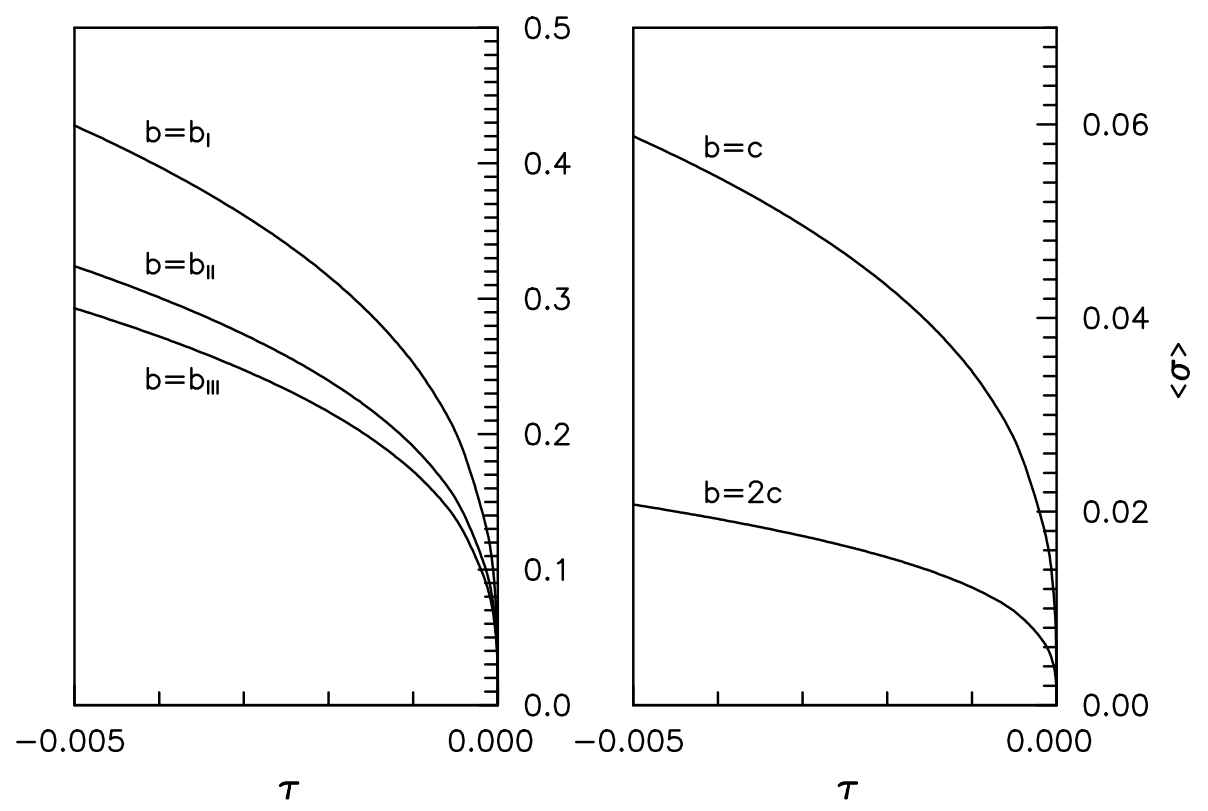

Figure 1. The temperature dependence of the average spin moment of the system in the $\rho^{6}$ model approximation for various values of the effective radius $b$ of the potential: $b_{\mathrm{I}}=c /(2 \sqrt{3}), b_{\mathrm{II}}=0.3379 c, b_{\mathrm{III}}=0.3584 c, c$, and $2 c$.

determining the coefficients of a microscopic analogue of the Landau free energy are given for various values of the interaction potential range.

Due to the factor $N$ in the exponent, the integrand in (7) possesses a sharp maximum at the point $\bar{\rho}$, which corresponds to the equilibrium value of the order parameter. The average spin moment playing the role of the order parameter for the investigated system is found.

The proposed method for computing a one-component spin system may be generalized to the case of a system with an $n$-component order parameter.

\section{References}

1. Yukhnovskii I.R. Phase Transitions of the Second Order. Collective Variables Method. Singapore, World Scientific, 1987.

2. Liu A.J., Fisher M.E. // Physica A, 1989, vol. 156, p. 35.

3. Landau D.P. // Physica A, 1994, vol. 205, p. 41.

4. Guida R., Zinn-Justin J. // J. Phys. A, 1998, vol. 31, p. 8103.

5. Pelissetto A., Vicari E. // Phys. Reports, 2002, vol. 368, p. 549.

6. Kozlovskii M.P., Pylyuk I.V. Calculation of the correlation length critical exponent of the three-dimensional Ising model with the use of non-Gaussian basis measures. - In: Proc. of the All-Union Conf. on Modern Problems in Statistical Physics. Vol. 2, Lviv, 3-5 February 1987, p. 50 (in Russian).

7. Kozlovskii M.P. // Teor. Mat. Fiz., 1989, vol. 78, p. 422 (in Russian).

8. Kozlovskii M.P., Pylyuk I.V. // Ukr. Fiz. Zh., 1990, vol. 35, p. 146 (in Ukrainian). 
9. Kozlovskii M.P., Pylyuk I.V., Dukhovii V.V. // Condens. Matter Phys., 1997, No. 11, p. 17.

10. Kozlovskii M.P., Pylyuk I.V., Dukhovii V.V. Calculation method for the threedimensional Ising ferromagnet thermodynamics within the frames of $\rho^{6}$ model. Preprint cond-mat/9907468, 1999, 41 p.

11. Yukhnovskii I.R., Kozlovskii M.P., Pylyuk I.V. // Z. Naturforsch., 1991, vol. 46a, p. 1.

12. Ma S. Modern Theory of Critical Phenomena. Reading, Massachusetts, Benjamin, 1976.

13. Pylyuk I.V. // Low Temp. Phys., 1999, vol. 25, p. 877.

14. Pylyuk I.V. // Low Temp. Phys., 1999, vol. 25, p. 953.

15. Yukhnovskii I.R., Kozlovskii M.P., Pylyuk I.V. Microscopic Theory of Phase Transitions in the Three-Dimensional Systems. Lviv, Eurosvit, 2001 (in Ukrainian).

16. Pylyuk I.V., Kozlovskii M.P. Thermodynamic characteristics of the 3D Ising system in $\rho^{6}$ model approximation taking into account the confluent correction. II. Lowtemperature region. Preprint of the Institute for Condensed Matter Physics, ICMP97-07U, Lviv, 1997, 32 p. (in Ukrainian).

17. Kozlovskii M.P., Pylyuk I.V., Usatenko Z.E. // Phys. Status Solidi (b), 1996, vol. 197, p. 465 .

\title{
Мікроскопічний аналог вільної енергії Ландау для тривимірних ізінгоподібних систем
}

\author{
М.П.Козловський, І.В.Пилюк, О.О.Притула \\ Інститут фізики конденсованих систем НАН України, \\ 79011 Львів, вул. Свєнціцького, 1 \\ Отримано 14 жовтня 2002 р., в остаточному вигляді - \\ 12 лютого 2003 р.
}

Мікроскопічний аналог вільної енергії Ландау для тривимірної однокомпонентної спінової системи знайдено нижче критичної температури в результаті прямих розрахунків. Отримані явні вирази дозволяють дослідити залежність коефіцієнтів аналогу від температури та мікроскопічних параметрів системи. На відміну від теорії Ландау температурна залежність цих коефіцієнтів $€$ неаналітичною. Величини, що визначають коефіцієнти у виразі для мікроскопічного аналогу вільної енергії Ландау, а також температурно залежні криві для параметра порядку системи, подані для різних значень радіуса ефективної дії експоненціально спадного потенціалу взаємодії.

Ключові слова: ізінгоподібна система, колективні змінні, вільна енергія Ландау, параметр порядку

PACS: $05.50 .+q, 64.60 . F r, 75.10 . H \mathrm{k}$ 